\title{
Correlation Inequalities for Ising Spin Lattices ${ }^{\star}$
}

\author{
J. K. Percus $\star \star$ \\ Courant Institute of Mathematical Sciences and Physics Department, \\ New York University, New York, N. Y., USA
}

Received October 22, 1974

\begin{abstract}
A change of spin representation is used to present expectation inequalities on Ising lattices directly as sums of terms of like sign. The technique is extended to correlation inequalities by introducing replica variables which convert correlations into expectations on a larger space. Second order correlations are analyzed in full from this viewpoint, recovering the FKG set, among others. Third order correlations are examined in some detail, and the sign of the multi-site Ursell correlations $F_{3}, F_{4}, F_{6}$ established under appropriate restrictions.
\end{abstract}

\section{Introduction}

Problems of substance in mathematical physics can rarely be solved exactly. Indeed, a large part of the field is devoted to developing approximation methods for dealing with otherwise intractable situations. However, the reliability of such methods is always suspect unless the magnitude of the error committed can at least be estimated. Still better, one can in increasingly many cases establish bounds

$$
A_{1} \leqq A \leqq A_{2}
$$

for the value of a desired quantity $A$. Since there is usually no inate applicable symmetry, very different methods may have to be used to establish upper and lower bounds. But, once established, such bounds can often be pyramided to bounding other quantities [1], comparing characteristics of similar systems [2], and the like.

It is the purpose of this paper to set up a suitable apparatus for bounding local observables in Ising spin lattices. We first review the relevant material concerning free energies, expectations, and correlations, then show how a change of representation can be an effective tool for establishing expectation inequalities. The replica variable formalism is introduced to extend this technique to correlations, and the problem of second order correlations fully analyzed within the bounds of the method. Finally, higher order Ursell correlations are introduced, and several are analyzed as part of the general investigation of higher order correlations.

$\star$ Based on talk given at Yeshiva University Statistical Mechanics Meeting, November, 1973.

$\star \star$ Supported in part by U. S. Atomic Energy Commission, Contract No. AT (11-1)-3077. 


\section{Energy Bounds}

Probably the most familiar nontrivial bounding principle is the Rayleigh-Ritz estimate of the ground state energy $E_{0}(H)$ for a quantum mechanical Hamiltonian $H$ :

$$
\begin{gathered}
E_{0}(H) \leqq\langle\phi|H| \phi\rangle \\
\text { where }\langle\phi \mid \phi\rangle=1,
\end{gathered}
$$

becoming an equality when $\phi$ is a correct ground state. As an alternative form,

$$
\begin{aligned}
& E_{0}(H) \leqq \operatorname{Tr} H \varrho \\
& \text { where } \varrho=|\phi\rangle\langle\phi|
\end{aligned}
$$

or more generally, where

$$
\operatorname{Tr} \varrho=1, \quad \varrho \text { positive semi-definite (p.s.d.). }
$$

Reverse inequalities can be obtained similarly, but tend to be very poor for systems of many degrees of freedom. If however the Hamiltonian is a symmetrized sum of sub-Hamiltonians, matters are greatly simplified [3]. Consider e.g. an $N$-particle system with at most pair interactions, so that

$$
\begin{aligned}
& H=\sum_{l \leqq i \leqq j \leqq N} H_{2}(i, j) \\
& \text { where } \quad H_{2}(i, j)=H_{2}(j, i) .
\end{aligned}
$$

Then for density matrices $\varrho$ symmetric in the particles, the ground state will be given by

$$
\begin{aligned}
E_{0}(H) & =\min _{\varrho} \operatorname{Tr} H \varrho \\
& =\operatorname{Min}_{\varrho}\left(\begin{array}{l}
N \\
2
\end{array}\right) \operatorname{Tr} H_{2}(1,2) \varrho \\
& =\operatorname{Min}_{\operatorname{Tr}_{3 \ldots N} \varrho \varrho_{2}}\left(\begin{array}{c}
N \\
2
\end{array}\right) \operatorname{Tr} H_{2}(1,2) \varrho_{2}(1,2),
\end{aligned}
$$

the minimization domain consisting of those two-body $\varrho_{2}$ which can be obtained by integrating out the remaining $N-2$ particles of some normalized positive semi-definite $\varrho$. If one subjects the minimization only to some necessary conditions on $\varrho_{2}$, the inequality becomes [4]

$$
\begin{aligned}
& E_{0}(H) \geqq\left(\begin{array}{c}
N \\
2
\end{array}\right) \operatorname{Min}_{\text {"@2" }} \operatorname{Tr} H_{2}(1,2) \varrho_{2}(1,2) \\
& \text { where " } \varrho_{2} " \text { means } \operatorname{Tr} \varrho_{2}=1, \varrho_{2} \text { p.s.d., } \varrho_{2}(12)=\varrho_{2}(21) \\
& \text { plus a selection of other necessary conditions. }
\end{aligned}
$$

The efficacy of the bound depends upon amassing a sufficient number of conditions which the pair density matrix $\varrho_{2}$ must satisfy.

At finite temperature, a similar situation obtains. For simplicity, consider here the classical domain, at fixed volume. Then the Helmholtz free energy at 
reciprocal temperature $\beta$ satisfies

$$
\begin{aligned}
& \begin{array}{l}
F(H) \leqq \int \varrho H-T S[\varrho] \\
\\
\quad=\int \varrho H+\frac{1}{\beta} \int \varrho \ln \varrho
\end{array} \\
& \text { where } \int \varrho=1, \varrho \geqq 0,
\end{aligned}
$$

achieving equality for the Gibbs state $\varrho=e^{-\beta H} / \int e^{-\beta H}$. For a reverse inequality, we may take a model Hamiltonian $H_{M}$ for which the free energy $F_{M}$ is known. Then

$$
\begin{aligned}
F(H) & =\operatorname{Min}_{\varrho} \int \varrho H-T S[\varrho] \\
\text { while } \quad F_{M} & \leqq \int \varrho H_{M}-T S[\varrho],
\end{aligned}
$$

so that

$$
F(H) \geqq F_{M}+\operatorname{Min}_{\varrho} \int \varrho\left(H-H_{M}\right) .
$$

If $H$ and $H_{M}$ are of the form (2.4), this reduces to [2]

$$
\begin{aligned}
& \quad F(H) \geqq F_{M}+\left(\begin{array}{c}
N \\
2
\end{array}\right) \text { Min“" } \varrho_{2}, \int \varrho_{2}\left(H_{2}-H_{2 M}\right) \\
& \text { where } \quad \int \varrho_{2}=1, \varrho_{2} \geqq 0, \varrho_{2}(12)=\varrho_{2}(21), \ldots
\end{aligned}
$$

Again a major problem is that of finding enough necessary conditions which $\varrho_{2}$ must satisfy.

\section{Bounds on Expectations}

Much more information on the structure of a system - and much more contact with experimental observation-is supplied by the expectations $\langle Q\rangle_{H}$ of ovservables in a specified state of a system of Hamiltonian $H$. Experimentally, these are often measured by energy changes under external stimuli. Theoretically as well, one has for classical or quantum systems the generalized susceptibility relation

$$
\langle Q\rangle_{H}=\left.\frac{\partial}{\partial \lambda} F(H+\lambda Q)\right|_{\lambda=0}
$$

so that all expectations can in principle be generated by free energies. Indeed expectations of $\delta$-functions can be used to extract the probability density or density matrix, so that all information on equilibrium system structure is in principle available this way.

Obtaining bounds on expectations is a good deal harder than the corresponding problem for energies, and a number of specialized techniques exist. Crucial to many of these is the convexity of the free energy under changes in a linear parameter: classically,

$$
\left(\partial^{2} / \partial \lambda^{2}\right) F(H+\lambda Q)=\left(\partial^{2} / \partial \lambda^{2}\right)(-1 / \beta) \ln \int e^{-\beta(H+\lambda Q)}=\beta\left(\langle Q\rangle_{H+\lambda Q}^{2}-\left\langle Q^{2}\right\rangle_{H+\lambda Q}\right),
$$

or

$$
\frac{\partial^{2}}{\partial \lambda^{2}} F(H+\lambda Q) \leqq 0
$$


with an identical result for the quantum mechanical case. An immediate consequence, one can see, is that for an approximate free energy lower bound which is exact at $\lambda=0$,

$$
\begin{aligned}
F_{A P}(H+\lambda Q) & \leqq F(H+\lambda Q) \\
F_{A P}(H) & =F(H),
\end{aligned}
$$

the corresponding value of $\langle Q\rangle_{H}$ is likewise a lower bound:

$$
\langle Q\rangle_{H}^{A P}=\left.\frac{\partial}{\partial \lambda} F_{A P}(H+\lambda Q)\right|_{\lambda=0} \leqq\left.\frac{\partial}{\partial \lambda} F(H+\lambda Q)\right|_{\lambda=0}=\langle Q\rangle_{H} .
$$

More generally, from

$$
\begin{aligned}
F(H+\lambda Q)= & F(H)+\lambda\langle Q\rangle_{H}+\frac{1}{2} \lambda^{2} F^{\prime \prime}\left(H+\lambda^{\prime} Q\right) \\
& \text { where } \quad 0 \leqq \lambda^{\prime} \leqq \lambda,
\end{aligned}
$$

we have $F(H+\lambda Q)-F(H) \leqq \lambda\langle Q\rangle_{H}$, so that $[5,6]$

$$
\begin{aligned}
& \langle Q\rangle_{H} \geqq \frac{1}{\lambda}[F(H+\lambda Q)-F(H)] \\
& \langle Q\rangle_{H} \leqq \frac{1}{\lambda}[F(H)-F(H-\lambda Q)],
\end{aligned}
$$

allowing $\langle Q\rangle_{H}$ to be bounded from above and below if $F$ is similarly bounded.

One can also proceed in opposite fashion and generate large classes of inequalities with the hope of bounding a particular expectation. Perhaps prototypical are the Hamiltonian independent relations [4]

$$
\left\langle c c^{*}\right\rangle \geqq 0
$$

for any $c$, and the Hamiltonian-dependent [7]

$$
\left\langle\left[A,\left[A^{*}, H\right]\right]\right\rangle \geqq 0,
$$

[] denoting the classical Poisson bracket. The latter is a direct result of the classical identity

or $[3,5]$

$$
0=\int\left[A, B e^{-\beta H}\right]=\int[A, B] e^{-\beta H}-\beta \int B[A, H] e^{-\beta H},
$$

$$
\beta\langle B[A, H]\rangle=\langle[A, B]\rangle ;
$$

to obtain (3.8), just choose $B=\left[A^{*}, H\right]$. The two inequalities (3.8) and (3.9) can also be combined and strengthened to a Bogoliubov-type inequality by using (3.9) as a reduction formula. Thus

or

$$
\begin{aligned}
\left\langle|c-\beta[A, H]|^{2}\right\rangle & =\left\langle c c^{*}\right\rangle+\beta \operatorname{Re}\left\langle\left(\beta\left[A^{*}, H\right]-2 c^{*}\right)[A, H]\right\rangle \\
& =\left\langle c c^{*}\right\rangle+\operatorname{Re}\left\langle\left[A, \beta\left[A^{*}, H\right]-2 c^{*}\right]\right\rangle \geqq 0,
\end{aligned}
$$

$$
\left\langle c c^{*}\right\rangle+\beta\left\langle\left[A,\left[A^{*}, H\right]\right]\right\rangle \geqq 2 \operatorname{Re}\left\langle\left[A, c^{*}\right]\right\rangle,
$$

an equality of course if $c=\beta[A, H]$. Extension to quantum systems is straightforward. 


\section{Correlation Inequalities}

Expectations give important qualitative information as well, depending mainly on their vanishing or not, and their sign if they do not vanish. [This mode of expression has of course largely psychological impact, for expectations of differences of projections, so viewed, can be used to generate all information about the system.] One such expectation of great importance in the context of this paper is that of long-range order: for a field-free $N$-site Ising spin system $(\sigma(i)= \pm 1$ at each site $i$ ) the long-range order is defined by

$$
x=\lim _{\mid i-j_{i} \rightarrow \infty} \lim _{N \rightarrow \infty}\langle\sigma(i) \sigma(j)\rangle,
$$

and it is known [8] that for sufficiently low temperature, $\gamma>0$ for the standard two-dimensional Ising model.

Understanding such qualitative properties really involves comparing them for systems whose differences are under control. Indeed the change of an expectation under a physical change brings in the topic of correlations. Again in the classical case, we have by direct computation

$$
\begin{aligned}
\frac{1}{\beta} \frac{\partial}{\partial \lambda}\langle R\rangle_{H-\lambda Q} & =\langle R Q\rangle-\langle R\rangle\langle Q\rangle \\
& =\langle(R-\langle R\rangle)(Q-\langle Q\rangle)\rangle \equiv F_{2}(Q, R),
\end{aligned}
$$

known as a second order correlation (here \langle\rangle always means \langle\rangle$_{H-\lambda Q}$ ), for $F_{2}(Q, R)$ also measures the degree of independence of $Q$ and $R$. In similar fashion

$$
\begin{aligned}
\frac{1}{\beta} \frac{\partial}{\partial \lambda} F_{2}(Q, R)_{H-\lambda P} & =\langle P Q R\rangle-\langle P\rangle\langle Q R\rangle-\langle Q\rangle\langle P R\rangle \\
& -\langle R\rangle\langle P Q\rangle+2\langle P\rangle\langle Q\rangle\langle R\rangle \\
& \equiv F_{3}(P, Q, R),
\end{aligned}
$$

the third order Ursell correlation or cumulant, involving products of three expectations.

As an example of the sort of correlation inequalities available, and of their utility, consider a field-free ferromagnetic Ising model:

$$
-\beta H=\Sigma J_{k l} \sigma(k) \sigma(l), \quad J_{k l} \geqq 0 .
$$

Then for comparison of long-range order, we would need the second order correlation

$$
\begin{aligned}
\frac{\partial\langle\sigma(i) \sigma(j)\rangle}{\partial J_{k l}}= & \langle\sigma(i) \sigma(j) \sigma(k) \sigma(l)\rangle \\
& -\langle\sigma(i) \sigma(j)\rangle\langle\sigma(k) \sigma(l)\rangle .
\end{aligned}
$$

Now according to Griffith's second inequality, [9]

$$
F_{2}(\sigma(i) \sigma(j), \sigma(k) \sigma(l)) \geqq 0,
$$

thus if the long-range order is positive, it remains positive when any of the couplings $J_{k l}$ is increased. For example, a 3-dimensional ferromagnetic Ising lattice can be created from a sheaf of plane lattices by turning up the intercoupling constants 
from $-\infty$. It therefore has long-range order over at least the parameter range of the plane model (if $i$ and $j$ are not in a common plane, a slight modification is needed).

Substantial theoretical questions are easily answerable in the same way, such as the existence of the thermodynamic limit $N \rightarrow \infty$ of expectations. The limit may be obtained by turning up the coupling with external sites, and this results in a monotonic change if the correlation with each $\sigma(k) \sigma(l)$ has a fixed sign. Since spin expectations are trivially bounded from above and below $(-1 \leqq \sigma \leqq 1)$, convergence is assured in such cases.

\section{Manifest Inequalities for Expectations}

There exist many classes of correlation inequalities and many techniques for their derivation. [3] Among classes, there are: kinematic inequalities, such as $\left\langle A^{+} A\right\rangle \geqq 0$, true for all states of all systems; model inequalities depending on the existence of a solvable model; dynamical inequalities depending explicitly on the Hamiltonian (Bogoliubov-type), or symmetries thereof, or special properties thereof (such as strict ferromagnetic interaction). Further, one can distinguish between inequalities on multilinear or other forms, such as the trivial, but valuable, positive semi-definiteness of the matrix $\left\langle A_{i}^{+} A_{j}\right\rangle-\left\langle A_{i}^{+}\right\rangle\left\langle A_{j}\right\rangle$, and point inequalities such as $\left\langle A_{i} A_{j}\right\rangle-\left\langle A_{i} A_{j}\right\rangle \geqq 0$ which are much more limited in their domain of validity and consequently often much more useful.

In all cases, methods of proof are available, ranging from: indirect, such as the usual Schwartz inequality proof; through semi-direct, such as demonstrating that $C(\lambda) \geqq 0$ for $\lambda \geqq 0$ because $C(0) \geqq 0$ and $\partial C(\lambda) / \partial \lambda \geqq 0$; on to direct or manifest: $C=\Sigma C_{\alpha} \geqq 0$ because each $C_{\alpha} \geqq 0$. It seems always possible to use the last form, and this is probably best as well for a systematic enumeration of possibilities. After the event, the direct method is rarely the simplest, but in view of the primitive nature of the domain we address ourselves to, it is the one we will employ. Indeed, for Ising systems or their extensions, we will find a rather surprising amount of information that may be obtained merely by a change of representation of the spin variables. We start with an easy example, the Griffiths first inequality [10], which nonetheless contains one basic concept of our later development.

Example 1. The Field-free Ferromagnetic Ising Model on $N$ sites. Here

$$
H=F-\Sigma J_{k l} \sigma(k) \sigma(l), \quad J_{k l} \geqq 0
$$

and the reference of the free energy $F$ is chosen so that the Gibbs density

$$
\varrho=\exp -\beta H=\exp \left(-\beta F+\Sigma \beta J_{k l} \sigma(k) \sigma(l)\right)
$$

is automatically normalized, in the sense that

$$
\frac{1}{2^{N}} \sum_{\{\sigma(i)= \pm 1\}} \varrho\left(\left\{\sigma_{i}\right\}\right)=1 .
$$

We now want to examine the expectation

$$
\langle\sigma(i) \sigma(j)\rangle=\frac{1}{2^{N}} \sum_{\{\sigma(i)= \pm 1\}} \sigma(i) \sigma(j) \exp \left(-\beta F+\Sigma \beta J_{k l} \sigma(k) \sigma(l)\right) .
$$


A more algebraic form is gotten by regarding the values \pm 1 of $\sigma(i)$ as diagonal elements of a diagonal matrix

$$
\sigma(i)=\left(\begin{array}{rr}
-1 & 0 \\
0 & 1
\end{array}\right)
$$

and converting the sum over their values to a trace of functions of this matrix. Indeed we can work in the product space of the $\{\sigma(i)\}$, so that

$$
\sigma(i) \rightarrow\left(\begin{array}{ll}
1 & 0 \\
0 & 1
\end{array}\right) \otimes\left(\begin{array}{ll}
1 & 0 \\
0 & 1
\end{array}\right) \otimes \cdots \otimes \sigma(i) \otimes \cdots \otimes\left(\begin{array}{ll}
1 & 0 \\
0 & 1
\end{array}\right)
$$

and the trace is then over the product space:

$$
\langle\sigma(i) \sigma(j)\rangle=\frac{1}{2^{N}} \operatorname{Tr} \sigma(i) \sigma(j) \exp \left(-\beta F+\Sigma \beta J_{k l} \sigma(k) \sigma(l)\right) .
$$

We will not distinguish between the $2 \times 2$ and $2^{N} \times 2^{N}$ matrices $\sigma(i)$ unless there is danger of confusion. Finally, on introducing the normalized trace

we have

$$
\operatorname{tr} \equiv \frac{1}{2^{N}} \operatorname{Tr}
$$

$$
\langle\sigma(i) \sigma(j)\rangle=\operatorname{tr} \sigma(i) \sigma(j) \exp \left(-\beta F+\Sigma \beta J_{k l} \sigma(k) \sigma(l)\right) .
$$

Now what is the virtue of expression (5.9) aside from brevity? It is that the evaluation depends only upon the algebraic and trace properties of the $\{\sigma(i)\}$. In fact, it is clear that the conditions

$$
\begin{aligned}
& \text { the } \sigma(i) \text { commute, } \sigma(i)^{2}=1, \operatorname{tr}(1)=1, \\
& \operatorname{tr} \prod_{i \in A} \sigma(i)=0 \text { when } A \subset(1, \ldots, N)
\end{aligned}
$$

can be used to reduce and evaluate any expression of the above form (5.9), independently of the representation we happen to choose. To prove the non-negativity of (5.9), we need only select the non-negative representation

$$
\sigma(i)=\left(\begin{array}{ll}
0 & 1 \\
1 & 0
\end{array}\right)
$$

for each spin. Doing so, we see that in the form

$$
\langle\sigma(i) \sigma(j)\rangle=\operatorname{tr} \sigma(i) \sigma(j)(\exp -\beta F)\left(\exp \Sigma \beta J_{k l} \sigma(k) \sigma(l)\right)
$$

the kernel $\sigma(i) \sigma(j)$ has non-negative matrix elements, $e^{-\beta F}$ is diagonal with nonnegative matrix elements, and the exponent $\Sigma \beta J_{k l} \sigma(k) \sigma(l)$ has non-negative matrix elements, implying the same for the exponential. In otjer words, we conclude that

$$
\langle\sigma(i) \sigma(j)\rangle \geqq 0
$$

because every term represented by the trace operation in (5.12) is non-negative. By a simple change of spin representation, we have created a manifest inequality. 


\section{Replica Variable Technique}

Let us proceed to the bounding of Ising model correlations. An nth order correlation contains terms of the form

$$
\begin{aligned}
& \prod_{\alpha=1}^{n}\left\langle G_{\alpha}(\boldsymbol{\sigma})\right\rangle=\prod_{\alpha=1}^{n}\left[\operatorname{tr} G_{\alpha}(\boldsymbol{\sigma}) e^{\phi(\boldsymbol{\sigma})}\right] \\
& \text { where } \boldsymbol{\sigma}=\{\sigma(i)\} \text { and } \phi(\boldsymbol{\sigma})=-\beta F-\beta H(\boldsymbol{\sigma}) .
\end{aligned}
$$

To deal with linear combinations of such products of expectations, at the very least a simplifying notation should be adopted, one which for example expresses the whole thing as a single expectation over a possibly larger space. In fact, this is precisely what one can do, as first pointed out in the Ising model context for $n=2$ by Ginibre [11]. Suppose we introduce a set of replicas $\boldsymbol{\sigma}_{1}, \boldsymbol{\sigma}_{2}, \ldots, \boldsymbol{\sigma}_{n}$ of $\boldsymbol{\sigma}$ and extend the trace operation to the $\left(2^{N}\right)^{n}$-dimensional product space. Thus we require the algebraic and trace relations

$$
\begin{aligned}
& \text { the } \sigma_{\alpha}(i) \text { commute, } \sigma_{\alpha}(i)^{2}=1, \operatorname{tr}(1)=1, \\
& \operatorname{tr} \prod_{(\alpha, i) \in A} \sigma_{\alpha}(i)=0 \text { for } A \subset\{(1,1), \ldots,(n, N)\} .
\end{aligned}
$$

Products of expectations are then indeed turned into expectations:

$$
\begin{aligned}
\prod_{\alpha=1}^{n}\left\langle G_{\alpha}(\boldsymbol{\sigma})\right\rangle & =\operatorname{tr} \prod_{\alpha=1}^{n} G_{\alpha}(\boldsymbol{\sigma}) e^{\phi\left(\boldsymbol{\sigma}_{\alpha}\right)} \\
& =\operatorname{tr} \prod_{\alpha=1}^{n} G_{\alpha}(\boldsymbol{\sigma}) \exp \sum_{\alpha=1}^{n} \phi\left(\boldsymbol{\sigma}_{\alpha}\right) .
\end{aligned}
$$

It now follows that any putative nth order correlation inequality can be written in the form

$$
\operatorname{tr} G\left(\sigma_{1}, \ldots, \sigma_{n}\right) e^{\sum_{1}^{n} \phi\left(\sigma_{\alpha}\right)} \geqq 0
$$

The direct method of proof would be to make each matrix element non-negative. If we want to be able to establish a number or a whole class of inequalities for a given physical system, i.e. for a given $\phi(\sigma)$, we are almost compelled to divide the task into two parts: first arrange matters so that the probability measure exp $\Sigma \phi\left(\sigma_{\alpha}\right)$ has non-negative elements, and second see which kernels $G$ have non-negative matrix elements. For the former, it is sufficient--but certainly not necessary - that

$$
\sum_{1}^{n} \phi\left(\sigma_{\alpha}\right)=\text { diagonal matrix }+ \text { non-negative matrix }
$$

(non-negative matrix meaning non negative matrix elements), for then [12]

$$
\exp \Sigma \phi\left(\boldsymbol{\sigma}_{\alpha}\right)=\lim _{t \rightarrow \infty}\left[\exp \left(\frac{1}{t} \operatorname{diag}\right) \exp \left(\frac{1}{t} \text { non-neg }\right)\right]^{t},
$$

as a product of non-negative diagonal matrices and non-negative matrices, is non-negative. We will hereafter insist on this condition, in the form

$$
\left(\sum_{1}^{n} \phi\left(\sigma_{\alpha}\right)\right)_{\text {non-diag }} \quad \text { is non-negative. }
$$


The hard part then consists of establishing the non-negativity of the proffered kernel.

It should be pointed out that the spin replica or "decoration" method [13] has appeared in a number of other contexts. A particularly valuable one is that of extending the domain of the independent variables from the Ising or spin $1 / 2$ case to arbitrary spin, or even continuous spin with given intrinsic probability distribution. For example [14], for spin $s, \sigma=\left(-\frac{s}{2}, 1-\frac{s}{2}, \ldots, \frac{s}{2}\right)$ with weight unity for each value, one can set

and, noting that at site $i$,

$$
\sigma(i)=\frac{1}{2} \sum_{q=1}^{s} \sigma_{q}(i)
$$

$$
\begin{gathered}
\sum_{\substack{s \\
1 \\
1} \sigma_{q}=M} \exp \left[\sum_{2}^{s} \frac{1}{2}\left(\sigma_{q-1} \sigma_{q}-1\right) \ln q\right] \\
=1 \text { for each value of } M,
\end{gathered}
$$

a dangling one-dimensional ferromagnetic Ising model at each vertex will guarantee a weight of unity.

\section{Second Order Correlations for Arbitrary External Field}

Consider now the simplest case, that of second order correlations. Hence we need only duplicate variables, which we denote by $\boldsymbol{\sigma}, \boldsymbol{\sigma}^{\prime}$. To start with, we shall have in mind only the bilinear ferromagnetic Ising model, but with arbitrary external field. In other words, the exponent of the Gibbs probability is given by

$$
\begin{gathered}
\phi(\sigma)=K+\Sigma u_{i} \sigma(i)+\Sigma v_{i j} \sigma(i) \sigma(j) \\
\text { where } \quad v_{i j} \geqq 0 .
\end{gathered}
$$

Then in order that $\left(\phi(\boldsymbol{\sigma})+\phi\left(\boldsymbol{\sigma}^{\prime}\right)\right)_{\text {non-diag }}$

$$
=\left(2 K+\Sigma u_{i}\left(\sigma(i)+\sigma^{\prime}(i)\right)+\Sigma v_{i j}\left(\sigma(i) \sigma(j)+\sigma^{\prime}(i) \sigma^{\prime}(j)\right)_{\text {non-diag }}\right.
$$

be non-negative for any set of $u_{i}$, we must have

$$
s=\frac{1}{2}\left(\sigma+\sigma^{\prime}\right)
$$

diagonal at each site. If we assume that $s(j)$ is still represented by the unit matrix at any site but $j$, then $s$ is a $4 \times 4$ matrix with eigenvalues $\frac{1}{2}( \pm 1 \pm 1)=-1,1,0,0$, so that we may choose

$$
s=\left(\begin{array}{r:lll}
-1 & & \\
& 1 & & \\
\hdashline-1 & 0 & \\
\hdashline & & 0
\end{array}\right)
$$


Next define

$$
d=\frac{1}{2}\left(\sigma-\sigma^{\prime}\right) .
$$

Since $s d=d s=\frac{1}{4}\left(\sigma^{2}-\sigma^{\prime 2}\right)=0$, then $d$ must appear as

$$
d=\left(\begin{array}{rll} 
& \\
& 1 & \\
- & d_{1} & d_{2} \\
& d_{3} & d_{4}
\end{array}\right)
$$

But $d$ also has eigenvalues $-1,1,0,0$ so that its non-vanishing sub-matrix has eigenvalues \pm 1 . Thus

Now

$$
\begin{gathered}
d_{1} d_{4}-d_{2} d_{3}=-1 \\
d_{4}=-d_{1} .
\end{gathered}
$$

$$
\phi(\boldsymbol{\sigma})+\phi\left(\boldsymbol{\sigma}^{\prime}\right)=2\left(K+\Sigma u_{i} s(i)+\Sigma \sigma_{i j}(s(i) s(j)+d(i) d(j))\right.
$$

and each $v_{i j} \geqq 0$ independently. Hence the final requirement emanating from (6.7) is that $d(i) d(j)$ have a non-negative non-diagonal part:

$$
(d \otimes d)_{\text {non-diag }} \quad \text { is non-negative. }
$$

The diagonal elements of $(d \otimes d)_{\alpha \alpha^{\prime} \beta \beta^{\prime}}=d_{\alpha \beta} d_{\alpha^{\prime} \beta^{\prime}}$ are those in which $\alpha=\beta$ and $\alpha^{\prime}=\beta^{\prime}$, and so (7.6) is to be augmented by

$$
\begin{aligned}
& d_{1} d_{2} \geqq 0, \quad d_{4} d_{2} \geqq 0 \\
& d_{1} d_{3} \geqq 0, \quad d_{4} d_{3} \geqq 0 \\
& d_{2}{ }^{2} \geqq 0, \quad d_{2} d_{3} \geqq 0, \quad d_{3}{ }^{2} \geqq 0 .
\end{aligned}
$$

Equations (7.6), (7.9) generate two possibilities.

(A) If $d_{1} \neq 0$ and hence $d_{4} \neq 0$, then (7.6b), (7.9a, b) imply that $d_{2}=0, d_{3}=0$ and so from (7.6a), $d_{1}=-1, d_{4}=1$ (or the reverse).

(B) If $d_{1}=d_{4}=0$, then from (7.6a) $d_{2} d_{3}=1$, and from (7.9c) $d_{2}$ and $d_{3}$ are real. We can choose $d_{2}=d_{3}=1$ without loss of generality.

Let us separately consider these two cases.

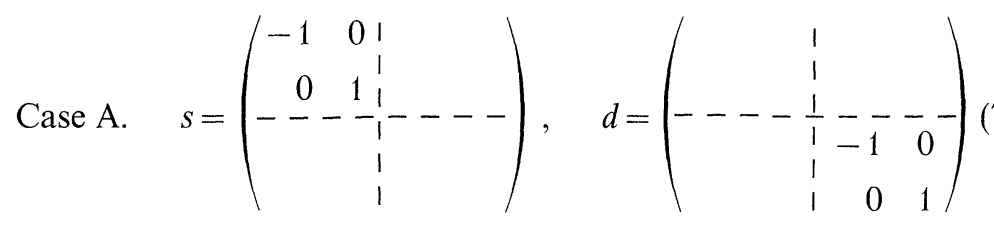

taken to hold at each site. At any site we then have

$$
\operatorname{tr}_{4} f(s, d)=\frac{1}{2}\left(\operatorname{tr}_{2} f(s, 0)+\operatorname{tr}_{2} f(0, d)\right),
$$

where the dimensionality of the space operated on by the trace is subscripted. Here, $s$ and $d$ take the canonical form (5.5) of Ising spins on complementary 
subspaces: $s=0$ if $d \neq 0, d=0$ if $s \neq 0$, and we can generalize (7.11) at once to

$$
\operatorname{tr} f(s, d)=\frac{1}{2^{N}} \sum_{A \in \Omega} \operatorname{tr}_{\{s(i) \mid i \in \bar{A}\}} \operatorname{tr}_{\{d(i) \mid i \in A\}} f(s, d) .
$$

Here $\Omega$ is the full set of sites $(1, \ldots, N)$, A is the index subset on which the $d(i)$ are spins, the $s(i)$ vanishing, and $\bar{A}$ the reverse. Compacting the notation in an obvious way, we conclude that

$$
\begin{aligned}
\operatorname{tr}\left[f\left(\boldsymbol{\sigma}, \boldsymbol{\sigma}^{\prime}\right) e^{\phi(\boldsymbol{\sigma})+\phi\left(\boldsymbol{\sigma}^{\prime}\right)}\right] & \\
= & \frac{1}{2^{N}} \sum_{A \subset \Omega} \operatorname{tr}_{s \in \bar{A}, d \in A}\left[f(\boldsymbol{s}+\boldsymbol{d}, \boldsymbol{s}-\boldsymbol{d}) e^{2 \sum v_{i j} d(i) d(j)}\right. \\
& \left.e^{2\left(K+\sum u_{i} S(i)+\sum v_{i j} s(i) s(j)\right.}\right] .
\end{aligned}
$$

Example 2. Two-Site Spin Correlations.

We consider $F_{2}(i, j)=\langle\sigma(i) \sigma(j)\rangle-\langle\sigma(i)\rangle\langle\sigma(j)\rangle=\frac{1}{2}\left\langle\sigma(i) \sigma(j)+\sigma^{\prime}(i) \sigma^{\prime}(j)-\sigma(i) \sigma^{\prime}(j)\right.$ $\left.-\sigma^{\prime}(i) \sigma(j)\right\rangle$, or

Hence (7.13) becomes

$$
F_{2}(i, j)=2\langle d(i) d(j)\rangle \text {. }
$$

$$
\begin{aligned}
F_{2}(i, j)= & \left.\frac{2}{2^{N}} \sum_{(i, j) \subset A \subset \Omega} \operatorname{tr}_{s \in \bar{A}} e^{2\left(K+\sum u_{i} s(i)+\sum v_{i j} s(i) s(j)\right.}\right] \\
& {\left[\operatorname{tr}_{d \in A} d(i) d(j) e^{2 \sum v_{i j} d(i) d(j)}\right] }
\end{aligned}
$$

or in terms of the two-site density

$$
\begin{gathered}
n_{2}(i, j) \equiv \Sigma\langle\sigma(i) \sigma(j)\rangle, \\
F_{2}(i, j \mid u, v, \beta, \Omega)=\sum_{(i, j) \subset A \subset \Omega} C_{A} n_{2}(i, j \mid 0, v, 2 \beta, A),
\end{gathered}
$$

Here $C_{A} \geqq 0$ for all $u, v$, and the external field $u$, interaction $v$, reciprocal temperature $\beta$, set of sites $A$ have all been indicated explicitly. We conclude in particular that if $n_{2}(i, j)$ at zero field has the same sign for the system on each sublattice containing $i$ and $j$, then this is the sign guaranteed $F_{2}(i, j)$ for the full lattice of sites in arbitrary external field at double the temperature. Thus the zero-field Griffiths' first inequality implies the second inequality at any field.

Example 3. Two-Site Spin Density.

On the basis of the above, it is worth reconsidering Example 1, Eq. (5.1) of the free-field two-site spin density. The transformation (7.15) offers some further insight into this quantity. At $u=0,(7.15)$ reduces to

$$
\begin{aligned}
n_{2}(i, j)= & \frac{e^{2 K}}{2^{N}} \sum_{(i j) \subset A \subset \Omega}\left[\operatorname{tr}_{s \in \bar{A}} e^{2 \sum v_{i j} s(i) s(j)}\right] \\
& {\left[\operatorname{tr}_{d \in A} 2 d(i) d(j) e^{2 \sum v_{i j} d(i) d(j)}\right] }
\end{aligned}
$$

Now $d(i)^{2} d(j)^{2}=1$ for $(i j) \subset A \subset \Omega$, so we have as well

$$
\begin{aligned}
\left\langle d(i)^{2} d(j)^{2}\right\rangle= & \frac{e^{2 K}}{2^{N}} \sum_{(i j) \subset A \subset \Omega}\left[\operatorname{tr}_{s \in \bar{A}} e^{2 \sum v_{i j} s(i) s(j)}\right] \\
& {\left[\operatorname{tr}_{d \in A} e^{2 \sum v_{i j} d(i) d(j)}\right] }
\end{aligned}
$$


But at 0 -field (so that $\langle\sigma(i)=\langle\sigma(j)\rangle=0$ ),

$$
\left\langle d(i)^{2} d(j)^{2}\right\rangle=\frac{1}{4}\left\langle\left(1-\sigma(i) \sigma^{\prime}(i)\right)\left(1-\sigma(j) \sigma^{\prime}(j)\right)\right\rangle=\frac{1}{4}\left(1+\langle\sigma(i) \sigma(j)\rangle^{2}\right) .
$$

Hence, suppressing the common pair $(i, j)$ but inserting the sublattice of sites and reciprocal temperature, $(7.18)$ can be rewritten as

$$
\begin{gathered}
\frac{2 n_{2}(\Omega \mid \beta)}{1+n_{2}(\Omega \mid \beta)^{2}}=\sum_{(i j) \subset A \subset \Omega} w(\Omega, A \mid 2 \beta) n_{2}(A \mid 2 \beta) \\
\text { with } \\
w(\Omega, A \mid 2 \beta)=\frac{\operatorname{tr}_{s \in \Omega-A} e^{2 \sum v_{i j} s(i) s(j)} \operatorname{tr}_{d \in A} e^{2 \sum v_{i j} d(i) d(j)}}{\sum_{(i j) \subset B \subset \Omega} \operatorname{tr}_{s \in \Omega-B} e^{2 \sum v_{i j} s(i) s(j)} \operatorname{tr}_{d \in A} e^{2 \sum v_{i j} d(i) d(j)}}
\end{gathered}
$$

a positive normalized (with respect to $A$ ) weight function, i.e. a positive Markov transition matrix [15]. We can easily iterate (7.20): adopting the convention that $w(\Omega, A)=0$ unless $(i j) \subset A \subset \Omega$, then

$$
\begin{aligned}
n_{2}(\Omega \mid \beta)= & \sum_{A_{1}, \ldots, A_{q}} \prod_{p=1}^{q}\left[\left(1+n_{2}\left(A_{p-1} \mid 2^{p-1} \beta\right) w\left(A_{p-1}, A_{p} \mid 2^{p} \beta\right)\right]\right. \\
& \cdot n_{2}\left(A_{q} \mid 2^{q} \beta\right)
\end{aligned}
$$

where $A_{0} \equiv \Omega$. The expression (7.22) can be seen to converge as $q \rightarrow \infty$, leading then to

$$
n_{2}(\Omega \mid \beta)=\sum_{A} W(\Omega, A \mid \beta) n_{2}(A \mid \infty)
$$

where $W(\Omega, A \mid \beta) \geqq 0$. We have thus shown that if a given two-site spin density has a common sign for all sublattices at zero temperature, then this sign is maintained at finite temperature.

\section{Extended Measures and Kernels}

We proceed to the representation given by

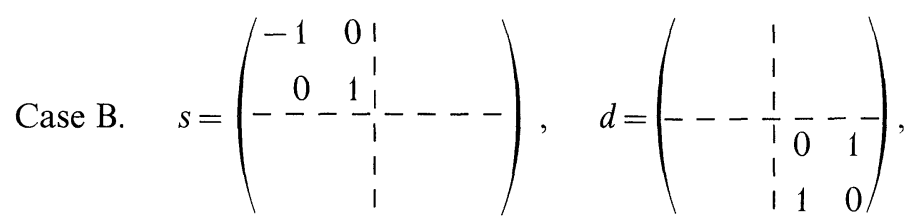

taken again to hold at each site. Since $d$ itself now has non-negative elements, this can be a considerably more useful tool, and we will push it to its limit. We first extend the domain of models by inquiring as to the largest class of Hamiltonians for which

$$
(\phi(s+d)+\phi(s-d))_{\text {non-diag }} \text { is non-negative }
$$

is guaranteed to hold. Now the non-vanishing elements of each $s(i)$ are wholly diagonal, those of each $d(i)$ wholly non-diagonal. Thus if $\phi$ is taken as multilinear in its arguments (a unique representation by virtue of $\sigma^{2}=1$ ), the diagonal part of $\phi(s+\boldsymbol{d})$ is precisely $\phi(s)$. Eq. (8.2) then becomes

$$
\phi(s+d)-2 \phi(s)+\phi(s-d) \quad \text { is non-negative, }
$$


or elementwise,

$$
\begin{gathered}
\phi\left(\left\{\begin{array}{r}
s(i) \mid i \in \bar{A} \\
1 \mid i \in A
\end{array}\right\}\right)-2 \phi\left(\left\{\begin{array}{r}
s(i) \mid i \in \bar{A} \\
0 \mid i \in A
\end{array}\right\}\right)+\phi\left(\left\{\begin{array}{c}
s(i) \mid i \in \bar{A} \\
-1 \mid i \in A
\end{array}\right\}\right) \geqq 0 \\
\text { where each } s(i)=+1 .
\end{gathered}
$$

To make contact with previous work, let us translate (8.4) into set language. We will denote by $\phi[T]$ the value of $\phi$ when $\sigma(i)=1$ for $i \in T, \sigma(i)=-1$ for $i \notin T$ :

$$
\phi[T] \equiv \phi\left(\left\{\begin{array}{l}
\sigma(i)=1 \mid i \in T \\
\sigma(i)=-1 \mid i \in \bar{T}
\end{array}\right\}\right) .
$$

Now choose, in (8.4), $C$ as the set on which $s(i)=1$ and eliminate the zero arguments by iterating the single site relation

$$
\phi(0)=\frac{1}{2}(\phi(1)+\phi(-1)) .
$$

Eq. (8.4) then becomes

$$
\phi[C+A]-\left(2 / 2^{A}\right) \sum_{B \subset A} \phi[C+B]+\phi[C] \geqq 0 .
$$

But (8.7) is clearly generated by its subcase in which $A$ consists of the pair of elements $(r, s) \subset \bar{C}$ :

$$
\phi[C+r+s]+\phi[C] \geqq \phi[C+r]+\phi[C+s]
$$

which in turn generates the more convenient extension

$$
\phi[A \cup B]+\phi[A \cdot B] \geqq \phi[A]+\phi[B] .
$$

The negative Hamiltonian or logarithm of the Gibbs measure is thus required to be convex as a set function-and all such Hamiltonians guarantee (8.2). The bilinear ferromagnetic $\phi$ of (7.1) is just a small, but crucial, subcase of this extensive FKG [16] class.

Next we inquire as to the full class of second order correlations which are manifestly non-negative by virtue of (8.1). The kernel $G\left(\sigma, \sigma^{\prime}\right)$ must therefore satisfy

$$
G(s+d, s-d) \quad \text { is non-negative. }
$$

For a given matrix element, let $A$ be the subset of sites at which $s=1, B$ at which $s=-1, K$ at which $d=1$, and $C$ at which $s=0$ and/or $d=0$, the four disjoint sets decomposing the full index set. Then if each triple argument denotes the subsets yielding the values $1,0,-1$ respectively, (8.10) becomes

$$
G[A+K, C, B ; A, C, B+K] \geqq 0 .
$$

Applying (8.6) and returning to the notation in which only the subset yielding the value +1 is indicated, (8.11) simplifies to

$$
\sum_{L \subset C, M \subset C} G[A+K+L ; A+M] \geqq 0,
$$

which is the desired set function restriction corresponding to (8.10). 
We should certainly translate (8.9) and (8.12) back into spin function language, and this can be done directly from (8.5) and easily enough for low order polynomials. Alternatively, if we have set functions with the required properties, then the spin functions are obtained by inverting (8.5):

$$
f(\boldsymbol{\sigma})=\sum_{A \subset \Omega} f[A] \prod_{i \in A}\left(\frac{1+\sigma(i)}{2}\right) \prod_{i \in \bar{A}}\left(\frac{1-\sigma(i)}{2}\right),
$$

as is readily verified. But the set function description is in fact closest to physical visualization: adding a site to a set merely means increasing its spin from -1 to +1 .

Example 4. Non-negative Ursell Correlations.

Let us consider a special case of (8.12), that in which

$$
G[A ; B]=f[A]-f[B] .
$$

Then (8.12) reduces to $\sum_{L \subset C}(f[A+K+L]-f[A+L]) \geqq 0$, equivalent to

$$
f[B+K] \geqq f[B]
$$

(if $K \cdot B=0$ ), i.e. $f[A]$ is a monotonic increasing set function. Of course $\left\langle f[A]-f\left[A^{\prime}\right]\right\rangle \geqq 0$ because it vanishes, but the real utility of $(8.15)$ is that any product of factors (8.14) will satisfy $(8.10)$ as well. For example, with only two factors, we have $\left\langle\left(f(\boldsymbol{\sigma})-f\left(\boldsymbol{\sigma}^{\prime}\right)\right)\left(g(\boldsymbol{\sigma})-g\left(\boldsymbol{\sigma}^{\prime}\right)\right)\right\rangle=2(\langle f(\boldsymbol{\sigma}) g(\boldsymbol{\sigma})\rangle-\langle f(\boldsymbol{\sigma})\rangle\langle g(\boldsymbol{\sigma})\rangle) \geqq 0$, i.e. $[16]$.

If $f(\sigma), g(\sigma)$ are non-decreasing as set functions on the sites of positive spin, then $F_{2}(f, g) \geqq 0$ for any Hamiltonian satisfying (8.9)

Example 5. Change of Observable under Finite Change of Hamiltonian According to (4.2), the result (8.16) can also be written as

$$
\left.\frac{\partial}{\partial \lambda}\langle g(\boldsymbol{\sigma})\rangle_{\phi(\boldsymbol{\sigma})+\lambda f(\boldsymbol{\sigma})}\right|_{\lambda=0} \geqq 0 .
$$

In fact, if $\phi(\sigma)+\lambda f(\boldsymbol{\sigma})$ for $\lambda \geqq 0$ were to satisfy (8.9) whenever $\phi(\boldsymbol{\sigma})$ does-which would occur e.g. if $f$ were a positive external field so that

$$
f[A \cup B]+f[A \cdot B]=f[A]+f[B]
$$

as well as (8.15)-then the restriction to $\lambda=0$ could be dropped:

$$
\frac{\partial}{\partial \lambda}\langle g(\sigma)\rangle_{\phi+\lambda f} \geqq 0 \quad \text { for } \quad \lambda \geqq 0 \text {. }
$$

We could then integrate over $\lambda$ from 0 to 1 , obtaining

$$
\langle g(\boldsymbol{\sigma})\rangle_{\phi+\lambda f} \geqq\langle g(\boldsymbol{\sigma})\rangle_{\phi} .
$$

(or the reverse inequality for decreasing function $f$ ). But the additional restriction (8.18). or even convexity, is not needed for (8.20); we have directly $\langle g\rangle_{\phi+f}-\langle g\rangle_{\phi}$ $=\left\langle g e^{f}\right\rangle_{\phi} /\left\langle e^{f}\right\rangle_{\phi}-\langle g\rangle_{\phi}=F_{2}\left(g, e^{f}\right) /\left\langle e^{f}\right\rangle \geqq 0$, since $e^{f}$ satisfies (8.15) when $f$ does. We can similarly compare two finite changes: if $F$ is the free energy, $F(\phi)=-\ln \operatorname{tr} e^{\phi}$, 
then $F(\phi+f)-F(\phi)=-\ln \left\langle e^{f}\right\rangle$ and $F(\phi+f+g)-F(\phi+f)-F(\phi+g)+F(\phi)$ $=-\ln \left(\left\langle e^{f+g}\right\rangle /\left\langle e^{f}\right\rangle\left\langle e^{g}\right\rangle\right) \leqq 0$, so that

$$
F(\phi+f+g)+F(\phi) \leqq F(\phi+f)+F(\phi+g)
$$

under conditions $(8.9,8.15)$ for $\phi, f, g$.

\section{Second Order Correlations for Negative Hamiltonians}

Equation (8.16), or its generalization to kernels of the form (8.12), applies to a well-delineated set of Hamiltonians, those for which (8.9) holds. For a more physical characterization of this class, we note first that imposition of an arbitrary external field, by virtue of (8.18), does not change the convexity of the measure. But by appending such a field, one can move the maximum or minimum of $\phi$ to any desired spin configuration. It is the behavior of $\phi$ with respect to its maximum or minimum which characterize the class in question. At least two facets of this behavior are easily ascertained.

Suppose first that $\phi[A]$ is a local minimum of $\phi$, i.e. with respect to single spin flips:

$$
\begin{aligned}
& \phi[A+b]-\phi[A] \geqq 0 \quad \text { for } \quad b \in \bar{A} \\
& \phi[A-c]-\phi[A] \geqq 0 \text { for } c \in A .
\end{aligned}
$$

Since Eq. (8.9), written as

$$
\begin{aligned}
& \phi[A+r+s]-\phi[A] \geqq(\phi[A+r)-\phi[A])+(\phi[A+s]-\phi[A]) \\
& \phi[A-r-s]-\phi[A] \geqq(\phi[A-r)-\phi[A])+(\phi[A-s)-\phi[A]),
\end{aligned}
$$

can be iterated to read

$$
\begin{aligned}
& \phi[A+B]-\phi[A] \geqq \sum_{b \in B}(\phi[A+b]-\phi[A]) \text { for } B \subset \bar{A} \\
& \phi[A-C]-\phi[A] \geqq \sum_{c \in C}(\phi[A-c]-\phi[A]) \text { for } C \subset A
\end{aligned}
$$

we see that (9.1) implies

$$
\begin{aligned}
& \phi[A] \leqq \phi[K] \\
& \text { when either } K \subset A \text { or } K \supset A,
\end{aligned}
$$

i.e. $\phi[A]$ remains a minimum when any set of +1 spins is switched to -1 , or vice versa. Next, suppose that $\phi[A]$ is a maximum with respect to any subset or superset:

$$
\phi[A] \geqq \phi[K]
$$

Now (8.9), in the form

$$
\text { for } K \subset A \text { or } K \supset A \text {. }
$$

$$
(\phi[A]-\phi[B]) \geqq(\phi[A]-\phi[A-B])+(\phi[A]-\phi[A \cup B]),
$$

shows that $\phi[A]$ is an absolute maximum.

Let us proceed to a rather different generalization of the ferromagnetic Ising model, that in which all coefficients present in $\phi(\sigma)$ are positive, i.e. all coefficients 
of $H[\sigma]$ are negative, and in particular the external field is negative or vanishing at each site. If we decompose $\phi(\sigma)$ into its monomial terms:

$$
\phi(\sigma)=\sum_{A \subset \Omega} \phi_{A} \prod_{i \in A} \sigma(i), \quad \phi_{A} \geqq 0
$$

then for each term

$$
\begin{aligned}
\prod_{i \in A} \sigma(i)+\prod_{i \in A} \sigma^{\prime}(i) & =\prod_{i \in A}(s(i)+d(i))+\prod_{i \in A}(s(i)-d(i)) \\
& =2 \sum_{B \subset A,|B| \text { even }}\left(\prod_{i \in A-B} s(i)\right)\left(\prod_{j \in B} d(j)\right)
\end{aligned}
$$

Thus $\phi(\boldsymbol{\sigma})+\phi\left(\boldsymbol{\sigma}^{\prime}\right)$ will certainly be non-negative if $s$ and $d$ are individually nonnegative. Without further ado, then, let us choose the representation

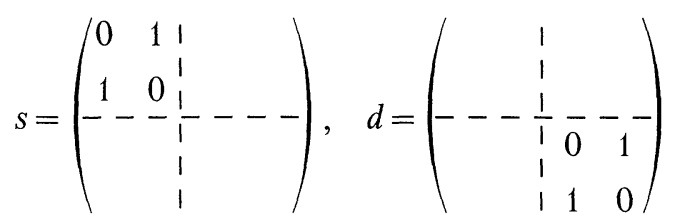

at each site, and thus guarantee a non-negative logarithmic measure for second order correlations of systems obeying (9.7).

Instead of aiming at full generality, let us now merely consider a large class of inequalities unavailable in the case of arbitrary convex $\phi$. For this purpose, we rewrite the kernel as

$$
G(s+d, s-d) \equiv \bar{G}(s, d) .
$$

Then clearly

$$
\bar{G}(s, d) \geqq 0 \quad \text { for } \quad s(i)=0,1, \quad d(i)=0,1
$$

will satisfy the matrix element relation

$$
G\left(\boldsymbol{\sigma}, \boldsymbol{\sigma}^{\prime}\right) \geqq 0
$$

Example 6. Non-negative Pair Correlations.

Suppose now that

$$
G(\boldsymbol{\sigma}, \boldsymbol{\sigma})=f^{\prime}(\boldsymbol{\sigma})-f\left(\boldsymbol{\sigma}^{\prime}\right)
$$

$f$ has non-negative coefficients.

For any monomial term, we have

$$
\prod_{i \in A}(s(i)+d(i))-\prod_{i \in A}(s(i)-d(i))=2 \sum_{B \subset A,|B| \text { odd }}\left(\prod_{i \in A-B} s(i)\right)\left(\prod_{j \in B} d(j)\right)
$$

so that (9.11) holds. Choosing $G\left(\boldsymbol{\sigma}, \boldsymbol{\sigma}^{\prime}\right)=\left(f(\boldsymbol{\sigma}) f\left(\boldsymbol{\sigma}^{\prime}\right)\right) \cdot\left(g(\boldsymbol{\sigma})-g\left(\boldsymbol{\sigma}^{\prime}\right)\right)$, it follows at once that [16]

$$
F_{2}(f(\boldsymbol{\sigma}), g(\boldsymbol{\sigma})) \geqq 0
$$

if $f$ and $g$ have non-negative coefficients.

Example 7. Fourth Order Spin Correlations at Zero Field. 
Let us advance one more step in the correlation function sequence (4.1), 4.3). We have

$$
\begin{aligned}
\frac{1}{\beta} \frac{\partial}{\partial \lambda} F_{2}(Q, R, S)_{H-\lambda P} & =\langle P Q R S\rangle-\langle P\rangle\langle Q R S\rangle-\langle Q\rangle\langle P R S\rangle-\langle R\rangle\langle P Q S\rangle \\
& -\langle S\rangle\langle P Q R\rangle-\langle P S\rangle\langle Q R\rangle-\langle P Q\rangle\langle R S\rangle-\langle P R\rangle\langle Q S\rangle \\
& +2\langle P Q\rangle\langle R\rangle\langle S\rangle+2\langle P R\rangle\langle Q\rangle\langle S\rangle+2\langle P S\rangle\langle Q\rangle\langle R\rangle \\
& +2\langle Q R\rangle\langle P\rangle\langle S\rangle+2\langle Q S\rangle\langle P\rangle\langle R\rangle+2\langle R S\rangle\langle P\rangle\langle Q\rangle \\
& -6\langle P\rangle\langle Q\rangle\langle R\rangle\langle S\rangle \equiv F_{4}(P, Q, R, S) .
\end{aligned}
$$

Suppose that $P, Q, R, S$ are four spins, say $\sigma(1), \sigma(2), \sigma(3), \sigma(4)$. Then if $\phi(\sigma)$ has only terms of even degree-so that in particular there is no external field-it is clear that $\langle\sigma(i)\rangle=\operatorname{tr}(\sigma(i) \exp \phi(\sigma))$ is the trace of an odd function and hence vanishes. The expression (9.16) thus becomes a second order correlation:

while

$$
\begin{aligned}
F_{4}(1,2,3,4)= & \langle\sigma(1) \sigma(2) \sigma(3) \sigma(4)\rangle-\langle\sigma(1) \sigma(2)\rangle\langle\sigma(3) \sigma(4)\rangle \\
& -\langle\sigma(1) \sigma(3)\rangle\langle\sigma(2) \sigma(4)\rangle-\langle\sigma(1) \sigma(4)\rangle\langle\sigma(2) \sigma(3)\rangle
\end{aligned}
$$

It can then be seen by direct substitution that

$F_{4}(1,2,3,4)+2 F_{2}(1,2) F_{2}(3,4)=4\langle s(1) s(2) d(3) d(4)+d(1) d(2) s(3) s(4)\rangle$,

and we conclude from $(9.11)$ that [17]

for non-positive even Hamiltonian

$$
F_{4}(1,2,3,4) \geqq-2 F_{2}(1,2) F_{2}(3,4)
$$

\section{Third Order Correlations}

We proceed to third order correlations, for which purpose a triplicate spin set $\sigma_{0}, \sigma_{1}, \sigma_{2}$ is required, and a further transformation to matrices of manifest positivity strongly suggested. Some preliminary remarks concerning possible transformations are in order. What we want to end up with is a convenient matric representation of the $2^{n}$-dimensional commutative algebra generated by $n$ spins, and the representation must then satisfy, at each site,

$$
\begin{gathered}
\sigma_{\alpha}^{2}=1, \quad \sigma_{\alpha} \sigma_{\beta}=\sigma_{\beta} \sigma_{\alpha} \\
\operatorname{Tr} 1=2^{n}, \quad \operatorname{Tr} \prod_{i \in A} \sigma_{\alpha}=0 \text { for } 0 \neq A \subset(1, \ldots n)
\end{gathered}
$$

This is to be done via a transformation to an auxiliary set $p_{1}, \ldots, p_{m}$

$$
\begin{gathered}
\sigma_{\alpha}=\xi_{\alpha}\left(\left\{p_{\beta}\right\}\right) \\
\text { where } \quad f_{\gamma}\left(\left\{p_{\beta}\right\}\right)=0, \quad \gamma=1, \ldots, c .
\end{gathered}
$$

On the algebraic side, we clearly must first demand that (10.1) be a consequence of (10.3) and (10.4). There remains (10.2). 
Suppose that we have a representation of $\left\{p_{\alpha}\right\}$ satisfying (10.4) in the form of a $2^{n}$-dimensional matrix algebra on a vector space of dimensionality $2^{n}$. Now the $2^{n}$ quantities

$$
Q_{A} \equiv \prod_{\alpha \in A}\left(\frac{1+\sigma_{\alpha}}{2}\right) \prod_{\beta \in \bar{A}}\left(\frac{1-\sigma_{\beta}}{2}\right)
$$

by virtue of (10.1), are commuting orthogonal idempotents:

$$
Q_{A} Q_{A}=\delta_{A, B} Q_{A}
$$

and certainly satisfy

$$
\operatorname{Tr} Q_{A}=n_{A}, \quad \text { a non-negative integer. }
$$

Further, since $\Sigma_{A} \mathrm{Q}_{A}=1$, then

$$
\sum_{A} \operatorname{Tr} Q_{A}=2^{n}
$$

We want to guarantee that

$$
Q_{A} \neq 0,
$$

for it would then follow that $\operatorname{Tr} Q_{A} \neq 0$, and from $(10.3,10.8)$ that

$$
\operatorname{Tr} Q_{s}=1 \text {. }
$$

Then indeed

$$
\prod_{\beta \in B} \sigma_{\beta}=\sum_{A}\left(\prod_{\beta \in B}(-1)^{|\beta \cdot \bar{A}|}\right) Q_{s}
$$

implies that (10.2) holds (half of the sets $A$ contain a given $\beta$, half do not). To guarantee $(10.9)$, it suffices that $(10.3,10.4)$ be invertible to read

$$
p_{\beta}=\varrho_{\beta}\left(\left\{\sigma_{\alpha}\right\}\right),
$$

for if any $Q_{A}$ were to vanish, the algebra of the $\left\{\sigma_{\alpha}\right\}$ would, by (10.11), be of dimension $<2^{n}$, and so therefore would be the algebra of the $p_{\beta}$. We conclude that [18]

If (10.3) and (10.4) imply (10.1), and (10.1) and (10.12) imply (10.4),

then any $2^{n}$-dimensional matrix algebra representation of the $\left\{p_{\alpha}\right\}$ generates a representation of the $\left\{\sigma_{\alpha}\right\}$ satisfying (10.2).

As a first step in securing a suitable representation of the triplicate spin set $\sigma_{0}, \sigma_{1}, \sigma_{2}$, let us introduce the combinations, at each site,

$$
\begin{aligned}
s & =\frac{1}{2}\left(\sigma_{0}+\sigma_{1}+\sigma_{2}\right) \\
d & =\frac{1}{2}\left(\sigma_{0}+\omega \sigma_{1}+\omega^{2} \sigma_{2}\right) \\
d^{\prime} & =\frac{1}{2}\left(\sigma_{0}+\omega^{2} \sigma_{1}+\omega \sigma_{2}\right),
\end{aligned}
$$

where $\omega=\exp 2 \pi i / 3$ is a primitive cube root of unity, with inverse

$$
\begin{aligned}
& \sigma_{0}=\frac{2}{3}\left(s+d+d^{\prime}\right) \\
& \sigma_{1}=\frac{2}{3}\left(s+\omega^{2} d+\omega d^{\prime}\right) \\
& \sigma_{2}=\frac{2}{3}\left(s+\omega d+\omega^{2} d^{\prime}\right) .
\end{aligned}
$$


The triplicate measure exponent $\phi\left(\sigma_{0}\right)+\phi\left(\sigma_{1}\right)+\phi\left(\sigma_{2}\right)$ will then be generated by linear combinations of terms of the type

$$
\begin{gathered}
\sigma_{0}+\sigma_{1}+\sigma_{2}=2 s \\
\sigma_{0}(1) \sigma_{0}(2)+\sigma_{1}(1) \sigma_{1}(2)+\sigma_{2}(1) \sigma_{2}(2)=\frac{4}{3}\left(s(1) s(2)+d(1) d^{\prime}(2)+d^{\prime}(1) d(2)\right) \\
\sigma_{0}(1) \sigma_{0}(2) \sigma_{0}(3)+\sigma_{1}(1) \sigma_{1}(2) \sigma_{1}(3)+\sigma_{2}(1) \sigma_{2}(2) \sigma_{2}(3) \\
=\frac{8}{9}\left[s(1) s(2) s(3)+d(1) d(2) d(3)+d^{\prime}(1) d^{\prime}(2) d^{\prime}(3)\right. \\
+s(1)\left(d(2) d^{\prime}(3)+d^{\prime}(2) d(3)\right)+s(2)\left(d(1) d^{\prime}(3)+d^{\prime}(1) d(3)\right) \\
\left.+s(3)\left(d(1) d^{\prime}(2)+d^{\prime}(1) d(2)\right)\right], \ldots
\end{gathered}
$$

Thus the general ferromagnetic case (9.7) could be handled if each of $s, d, d^{\prime}$ had a non-negative representation. This however cannot be.

For a better idea of possible representations, let us make a further transformation to variables $c, d$. From (10.1), it is easy to see that

$$
\begin{aligned}
& \text { if } \quad c=\frac{1}{4}\left(\sigma_{0}+\sigma_{1}+\sigma_{2}+\sigma_{0} \sigma_{1} \sigma_{2}\right), \\
& d=\frac{1}{2}\left(\sigma_{0}+\omega \sigma_{1}+\omega^{2} \sigma_{2}\right) \\
& \text { then } \quad c^{2}+d^{6}=1 \quad c d=0 .
\end{aligned}
$$

Furthermore, (10.17) are invertible to read

$$
s=\frac{3}{2} c-\frac{1}{2} d^{3}, \quad d^{\prime}=d^{5} .
$$

We therefore need only an 8-dimensional representation of the algebra of $c, d$. Since $s d=-\frac{1}{2} d^{4}$ foilows from (10.17), and $s d \neq 0$, there is no non-negative representation of both $s$ and $d$. However, we certainly can choose

$$
s \geqq 0, \quad d \leqq 0, \quad d^{\prime} \leqq 0,
$$

e.g. by taking $c$ and $d$ in the form

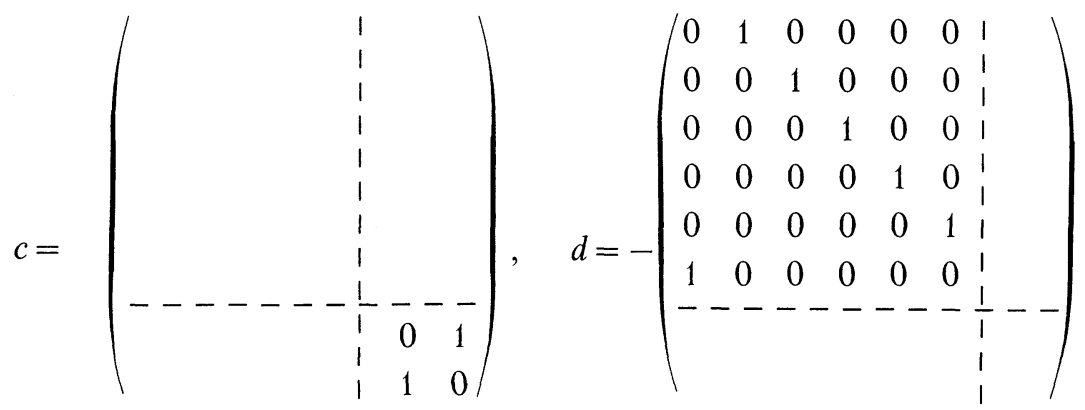

Then at least

$$
\phi\left(\boldsymbol{\sigma}_{0}\right)+\phi\left(\boldsymbol{\sigma}_{1}\right)+\phi\left(\boldsymbol{\sigma}_{2}\right) \geqq 0 \quad \text { for } \quad \phi(\boldsymbol{\sigma})=\Sigma u_{i} \sigma(i)+\Sigma v_{i j} \sigma(i) \sigma(j),
$$

so the triplet measure for the basic ferromagnetic Hamiltonian is non-negative.

Example 8. Third Order Spin Correlations at Negative Field. 
The Ursell function for spins at three sites

$$
\begin{aligned}
F_{2}(1,2,3)= & \langle\sigma(1) \sigma(2) \sigma(3)\rangle-\langle\sigma(1)\rangle\langle\sigma(2) \sigma(3)\rangle \\
& -\langle\sigma(2)\rangle\langle\sigma(1) \sigma(3)\rangle-\langle\sigma(3)\rangle\langle\sigma(1) \sigma(2)\rangle \\
& +2\langle\sigma(1)\rangle\langle\sigma(2)\rangle\langle\sigma(3)\rangle
\end{aligned}
$$

is most easily considered via the generating function

$$
\begin{aligned}
F_{3}[h] & \equiv \Sigma F_{3}(i, j, k) h(i) h(j) h(k) \\
& =\left\langle(\boldsymbol{h} \cdot \boldsymbol{\sigma})^{3}-3\langle\boldsymbol{h} \cdot \boldsymbol{\sigma})^{2}\right\rangle\langle\boldsymbol{h} \cdot \boldsymbol{\sigma}\rangle+2\langle\boldsymbol{h} \cdot \boldsymbol{\sigma}\rangle^{3} \\
& =\left\langle(\boldsymbol{h} \cdot \boldsymbol{\sigma}-\langle\boldsymbol{h} \cdot \boldsymbol{\sigma}\rangle)^{3}\right\rangle,
\end{aligned}
$$

or in triplicate spin notation $(i, j)=0,1,2)$

$$
\begin{aligned}
F_{3}(h) & =\left\langle\frac{1}{3} \Sigma\left(\boldsymbol{h} \cdot \boldsymbol{\sigma}_{i}\right)^{3}-\frac{1}{2} \sum_{i \neq j}\left(\boldsymbol{h} \cdot \boldsymbol{\sigma}_{i}\right)^{2}\left(\boldsymbol{h} \cdot \boldsymbol{\sigma}_{j}\right)+\frac{1}{3} \sum_{i \neq j \neq k}\left(\boldsymbol{h} \cdot \boldsymbol{\sigma}_{i}\right)\left(\boldsymbol{h} \cdot \boldsymbol{\sigma}_{j}\right)\left(\boldsymbol{h} \cdot \boldsymbol{\sigma}_{k}\right)\right\rangle \\
& =\left\langle\frac{3}{2} \Sigma\left(\boldsymbol{h} \cdot \boldsymbol{\sigma}_{i}\right)^{3}-\frac{3}{2} \Sigma\left(\boldsymbol{h} \cdot \boldsymbol{\sigma}_{i}\right)^{2}\left(\Sigma \boldsymbol{h} \cdot \boldsymbol{\sigma}_{j}\right)+\frac{1}{3} \Sigma \boldsymbol{h} \cdot \boldsymbol{\sigma}_{i} \Sigma \boldsymbol{h} \cdot \boldsymbol{\sigma}_{j} \Sigma \boldsymbol{h} \cdot \sigma_{k}\right\rangle .
\end{aligned}
$$

Inserting (10.15), we find at once

$$
\begin{aligned}
F_{3}[h] & =\frac{4}{9}\left\langle(\boldsymbol{h} \cdot \boldsymbol{d})^{3}+\left(\boldsymbol{h} \cdot \boldsymbol{d}^{\prime}\right)^{3}\right\rangle \\
& =\frac{8}{9}\left\langle(\boldsymbol{h} \cdot \boldsymbol{d})^{3}\right\rangle,
\end{aligned}
$$

and so conclude (GHS [17, 19] that for bilinear ferromagnetic spin system with negative external field, $F_{3}(1,2,3) \leqq 0$. By reversing the signs of all spins, it follows as well that $F_{3}(1,2,3) \geqq 0$ for positive external field.

\section{Higher Ursell Functions}

In order to proceed to higher order correlations, a concise representation of such functions is mandatory. Suppose that we want to examine the Ursell correlations or cumulants of the set of observables $f_{\alpha}, \alpha=1, \ldots$, . These are iteratively defined, in terms of the exponent

by (see $4.2,4.3,9.16)$

$$
\phi=-\beta H,
$$

$$
\begin{aligned}
& F_{n}\left(f_{1}, \ldots, f_{n-1}, f_{n}\right)_{\phi} \\
& \quad=\left.\frac{\partial}{\partial \lambda} F_{n-1}\left(f_{1}, \ldots, f_{n-1}\right)_{\phi+\lambda f_{n}}\right|_{\lambda=0},
\end{aligned}
$$

and hence directly by

$$
F_{n}\left(f_{1}, \ldots, f_{n}\right)_{\phi}=-\left.\left(\prod_{\alpha=1}^{n} \frac{\partial}{\partial \lambda_{\alpha}}\right) F\left[\phi+\sum_{1}^{n} \lambda_{\alpha} f_{\alpha}\right]\right|_{\left\{\lambda_{\alpha}=0\right\}},
$$

in terms of the suitably normalized free energy

$$
F[\phi]=-\ln \operatorname{tr} e^{\phi} .
$$


The generating function representation

$$
\begin{aligned}
& \sum_{\alpha=1}^{\infty}\left(\prod_{i=1}^{n} h_{\alpha_{1}}\right) F_{n}\left(\cdots f_{\alpha_{2}} \cdots\right)_{\phi} \\
& =F_{n}\left(\Sigma h_{\alpha} f_{\alpha}, \ldots, \Sigma h_{\alpha} f_{\alpha}\right)_{\phi} \\
& =-\left.\left(\frac{\partial}{\partial \lambda}\right)^{n} F\left[\phi+\lambda \Sigma h_{\alpha} f_{\alpha}\right]\right|_{\lambda=0}
\end{aligned}
$$

follows at once. Thus defining

we see finally that

$$
F_{n}[f] \equiv F_{n}(f, f, \ldots, f),
$$

$$
\begin{aligned}
\sum_{1}^{\infty} \frac{\lambda^{n}}{n !} F_{n}[f] & =\ln \operatorname{tr} e^{\phi+\lambda f}-\ln \operatorname{tr} e^{\phi} \\
& =\ln \left\langle e^{\lambda f}\right\rangle .
\end{aligned}
$$

The basic representation (11.7) can be used to write down $F_{n}[f]$ iteratively, or explicitly, or to quickly find a number of its properties. $F_{n}[f]$ is clearly an integral rational function of the $\left\langle f^{s}\right\rangle$. Further since $\lambda^{n} F_{n}[f]=F_{n}[\lambda f]$, it is homogeneous of degree $n$. Most importantly, however, suppose that $f$ and $f^{\prime}$ are independent variables: $\left\langle a(f) b\left(f^{\prime}\right)\right\rangle=\langle a(f)\rangle\left\langle b\left(f^{\prime}\right)\right\rangle$. Then $\ln \left\langle e^{\lambda\left(f+f^{\prime}\right)}\right\rangle$ $=\ln \left\langle e^{\lambda f}\right\rangle\left\langle e^{\lambda f^{\prime}}\right\rangle=\ln \left\langle e^{\lambda f}\right\rangle+\ln \left\langle e^{\lambda f^{\prime}}\right\rangle$, or $F_{n}\left[f+f^{\prime}\right]=F_{n}[f]+F_{n}\left[f^{\prime}\right]$. There is also at least a constant of proportionality to be applied. This may be found by choosing a special distribution for $f\left(e^{-f}\right.$ works very easily) or simply by noting the leading term of $F_{n}[f]$. We conclude that
a. The Ursell function $F_{n}[f]$ is an integral rational function of the $\left\langle f^{s}\right\rangle$
b. $F_{n}[\lambda f]=\lambda^{n} F_{n}[f]$
c. $F_{n}\left[f+f^{\prime}\right]=F_{n}[f]+F_{n}\left[f^{\prime}\right]$ for independent $f$ and $f^{\prime}$
d. $F_{n}[f]=\left\langle f^{n}\right\rangle+G_{n}\left[\langle f\rangle, \ldots,\left\langle f^{n-1}\right\rangle\right]$.

There are various ways of showing that (11.8) uniquely specifies the expression $F_{n}[f]$. For example, write

$$
\mu_{s} \equiv\left\langle f^{s}\right\rangle, \quad v_{s} \equiv\left\langle f^{\prime s}\right\rangle, \quad s=1, \ldots, n .
$$

Then, since $\left\langle\left(f+f^{\prime}\right)^{s}\right\rangle=\sum_{q}\left(\begin{array}{l}s \\ q\end{array}\right) \mu_{s-q} v_{q},(11.8 \mathrm{c})$ becomes

$$
\begin{aligned}
F_{n}\left\{\ldots, \sum_{q}\left(\begin{array}{l}
s \\
q
\end{array}\right) \mu_{s-q} v_{q}, \ldots\right\} & \\
& =F_{n}\left\{\ldots, \mu_{s}, \ldots\right\}+F_{n}\left\{\ldots, v_{s}, \ldots\right\} .
\end{aligned}
$$

Now applying the operation $\partial /\left.\partial v_{q}\right|_{f^{\prime}=0}$,

$$
\sum_{s}\left(\begin{array}{l}
s \\
q
\end{array}\right) \mu_{s-q} \frac{\partial F_{n}}{\partial \mu_{s}}=\left.\frac{\partial F_{n}}{\partial \mu_{s}}\right|_{f=0}, \quad q=1, \ldots, n .
$$


If we insert (11.8d) and include (11.8a) only as a boundary condition, (11.8) becomes equivalent to

$$
\begin{aligned}
& \left.a^{\prime} \cdot F_{n}\right|_{f=0}=0 \\
& b^{\prime} \cdot \sum_{s}\left(\begin{array}{l}
s \\
q
\end{array}\right) \mu_{s-q} \frac{\partial F_{n}}{\partial \mu_{s}}=\delta_{n, q},
\end{aligned}
$$

integrable, solvable for the $\partial \mathrm{F}_{n} / \partial \mu_{s}$, and so prossessing a unique solution.

On the basis of (11.8), the replica variable technique can be used to obtain a number of useful forms for $F_{n}$. The beginning of one class of forms has already been seen, i.e. from (7.14) and (10.25)

$$
\begin{aligned}
& F_{2}[\boldsymbol{h} \cdot \boldsymbol{\sigma}]=\frac{1}{2}\left\langle\left(\boldsymbol{h} \cdot \boldsymbol{\sigma}-\boldsymbol{h} \cdot \boldsymbol{\sigma}^{\prime}\right)^{2}\right\rangle \\
& F_{3}[\boldsymbol{h} \cdot \boldsymbol{\sigma}]=\frac{1}{3}\left\langle\left(\boldsymbol{h} \cdot \boldsymbol{\sigma}_{0}+\omega \boldsymbol{h} \cdot \boldsymbol{\sigma}_{1}+\omega^{2} \boldsymbol{h} \cdot \boldsymbol{\sigma}_{2}\right)^{3}\right\rangle .
\end{aligned}
$$

This suggests investigating functions of single linear combinations of the replica variables. Consider then for fixed $p \geqq n$,

$$
P_{n}[f]=K\left\langle\left(\sum_{1}^{p} \omega_{\alpha} f_{\alpha}\right)^{n}\right\rangle \text {. }
$$

$P_{n}[f]$ of course satisfies $(11.8 \mathrm{a}, \mathrm{b})$; it also satisfies $(11.8 \mathrm{~d})$ if $K=1 /\left(\sum_{1}^{p} \omega_{\alpha}^{n}\right)$. Can $P_{n}[f]$ satisfy $(11.8 \mathrm{c})$ as well? Clearly we have

$$
P_{n}\left[f+f^{\prime}\right]=K \sum_{q=0}^{n}\left(\begin{array}{l}
n \\
q
\end{array}\right)\left\langle\left(\sum_{1}^{p} \omega_{\alpha} f_{\alpha}\right)^{n-q}\right\rangle\left\langle\left(\sum_{1}^{p} \omega_{\alpha} f_{\alpha}^{\prime}\right)^{q}\right\rangle
$$

$P_{n}\left[f+f^{\prime}\right]$ will therefore reduce to $P_{n}[f]+P_{n}\left[f^{\prime}\right]$ if only the terms $q=0$ and $q=n$ are present, which holds if

$$
\left\langle\left(\sum_{1}^{p} \omega_{\alpha} f_{\alpha}\right)^{s}\right\rangle=0 \text { for } s \leqq n / 2
$$

for arbitrary $f$. But $\left\langle\prod_{1}^{p} f_{\alpha}^{s_{\alpha}}\right\rangle$ has the same value for any permutation of the $f$, so that (11.16) is equivalent to

$$
\sum_{\text {Perm. }} \prod_{1}^{p} \omega_{\alpha}^{s_{\alpha}}=0 \quad \text { whenever } \quad \Sigma s_{\alpha}=s \leqq n / 2
$$

and this in turn to

or

$$
\sum_{1}^{p} \omega_{\alpha}^{t}=0 \quad \text { for } \quad 0<t \leqq n / 2,
$$

$$
C_{t}\left\{\omega_{\alpha}\right\}=0 \text { for } 0 \leqq t \leqq n / 2 \text {, }
$$

$C_{t}$ being the $t^{\text {th }}$ elementary symmetric function of $\omega_{1}, \ldots, \omega_{p}$. 
Summarizing then,

$$
\begin{aligned}
& \text { if } C_{t}\left\{\omega_{\alpha}\right\}=0 \text { for } 0<t \leqq n / 2 \\
& \text { and } \quad \sum_{1}^{p} \omega_{\alpha}^{n} \neq 0 \\
& \text { then } \quad F_{n}[f]=\left\langle\left(\sum_{1}^{p} \omega_{\alpha} f_{\alpha}\right)^{n}\right\rangle / \sum_{1}^{p} \omega_{\alpha}^{n} .
\end{aligned}
$$

In the special case $p=n, \omega_{\alpha}=\omega^{\alpha}$, where $\omega=e^{2 \pi i / n}$ is a primitive $n$th root of unity, so that $\omega_{\alpha}^{n}-1=0$, we have $C_{t}=0$ for $0<t<n$. Thus

$$
\begin{aligned}
& F_{n}[f]=\frac{1}{n}\left\langle\left(\sum_{1}^{n} \omega^{\alpha} f_{\alpha}\right)^{n}\right\rangle \\
& \text { where } \quad \omega=e^{2 \pi i / n} .
\end{aligned}
$$

Equations (11.13) constitute cases $n=2,3$ and the general expression (11.21) was obtained by Cartier [20]. One disadvantage of (11.21) is that, oddly enough, an nth order replica system can be used to treat $F_{n}$, but not any lower Ursell function. This can be avoided by using only condition (11.20) and not extending it to $0<t<n$.

Example 9. Simultaneous Inequalities for $F_{2}$ and $F_{3}$.

Consider the triplicate system $p=3: \sigma_{0}, \sigma_{1}, \sigma_{2}$ and the corresponding generators $f_{\alpha}=\boldsymbol{h} \cdot \boldsymbol{\sigma}_{\alpha}$. If we choose $\omega_{0}=2, \omega_{1}=-1, \omega_{2}=-1$, then (11.20) is satisfied for $n=2, n=3$ :

$$
\begin{aligned}
& F_{2}[f]=\left\langle\left(2 f_{0}-f_{1}-f_{2}\right)^{2}\right\rangle / 6 \\
& F_{3}[f]=\left\langle\left(2 f_{0}-f_{1}-f_{2}\right)^{3}\right\rangle / 6 .
\end{aligned}
$$

But in the notation of (10.14),

$$
2 f_{0}-f_{1}-f_{2}=2 \boldsymbol{h} \cdot\left(\boldsymbol{d}+\boldsymbol{d}^{\prime}\right),
$$

which is non-positive in the representation (10.20) - by which we mean that, as a function of $\boldsymbol{h}$, all coefficients are non-positive. Thus, non-negativity of $F_{2}(1,2)$ and nonpositivity of $F_{3}(1,2,3)$ for negative field bilinear ferromagnetic lattices are again est ablished.

The situation at zero field is even simpler. Suppose more generally that the Hamiltonian is an even spin function, possessing only terms of even degree. And, rather than correlating single spins, suppose we merely require the correlated quantities to be odd spin functions. Thus, we want to consider $F_{n}[f]$ for odd $f$, where $\left\langle f^{2 p+1}\right\rangle=0$. Under these circumstances, $F_{n}[f]=0$ for odd $n$, so only $F_{2 n}[f]$ is of interest. But now

$$
\begin{aligned}
\left\langle\left(\sum_{1}^{p} \omega_{\alpha}\left(f_{\alpha}+f_{\alpha}^{\prime}\right)\right)^{2 n}\right\rangle & =\Sigma\left(\begin{array}{c}
2 n \\
q
\end{array}\right)\left\langle\left(\sum_{1}^{p} \omega_{\alpha} f_{\alpha}\right)^{2 n-q}\right\rangle\left\langle\left(\sum_{1}^{p} \omega_{\alpha} f_{\alpha}^{\prime}\right)^{q}\right\rangle \\
& =\Sigma\left(\begin{array}{c}
2 n \\
2 q
\end{array}\right)\left\langle\left(\sum_{1}^{p} \omega_{\alpha} f_{\alpha}\right)^{2 n-2 q}\right\rangle \cdot\left\langle\left(\sum_{1}^{p} \omega_{\alpha} f_{\alpha}^{\prime}\right)^{2 q}\right\rangle
\end{aligned}
$$

reduces to its first and last terms alone if

$$
\left\langle\left(\sum_{1}^{p} \omega_{\alpha} f_{\alpha}^{\prime}\right)^{2 q}\right\rangle=0 \quad \text { for } \quad 0<q \leqq n / 2
$$


or

$$
\sum_{\text {Perm }} \prod_{1}^{p} \omega_{\alpha}^{2 q_{\alpha}}=0 \text { for } 0<\Sigma q_{\alpha}=q \leqq n / 2,
$$

a condition on the elementary symmetric functions of the $\omega_{\alpha}^{2}$. Hence, accompanying (11.20) we have

$$
\begin{aligned}
& \text { if } C_{t}\left\{\omega_{\alpha}{ }^{2}\right\} \text { for } 0<t \leqq n / 2 \\
& \text { and } \sum_{1}^{p} \omega_{\alpha}{ }^{2 n} \neq 0 \\
& \text { then } F_{2 n}[f]=\left\langle\left(\sum_{1}^{p} \omega_{\alpha} f_{\alpha}\right)^{2 n}\right\rangle / \sum_{1}^{p} \omega_{\alpha}{ }^{2 n}
\end{aligned}
$$

when $f$ is an odd spin function

and the Hamiltonian is an even spin function.

Example 10. Sign of $F_{4}$ and $F_{6}$ at Zero Field.

To examine $F_{4}$, we need $n=2$ in (11.26), and so $\omega_{1}{ }^{2}+\omega_{2}{ }^{2}=0, \omega_{1}{ }^{4}+\omega_{2}{ }^{4} \neq 0$. We may choose, for example, $\omega_{1}$ and $\omega_{2}$ as conjugate fourth roots of -1 :

$$
\omega_{1}=e^{2 \pi i / 8}, \quad \omega_{2}=e^{-2 \pi i / 8} .
$$

Correspondingly, let us set

$$
p=\omega_{1} \sigma+\omega \sigma^{\prime}, \quad p^{\prime}=\omega_{2} \sigma+\omega_{1} \sigma^{\prime},
$$

satisfying the algebraic relations

$$
p^{2}=p^{\prime 2}, \quad p^{4}=4 \text {. }
$$

Then $\sigma^{2}=\sigma^{\prime 2}=1$ indeed follows from (11.29) and the inverse of (11.28),

$$
\sigma=\frac{1}{2}\left(\omega_{2} p+\omega_{1} p^{\prime}\right), \quad \sigma^{\prime}=\frac{1}{2}\left(\omega_{1} p+\omega_{2} p^{\prime}\right) .
$$

Furthermore,

Since

$$
\sigma(1) \sigma(2)+\sigma^{\prime}(1) \sigma^{\prime}(2)=\frac{1}{2}\left(p(1) p^{\prime}(2)+p^{\prime}(1) p(2)\right)
$$

$$
F_{4}[\boldsymbol{h} \cdot \boldsymbol{\sigma}]=-\frac{1}{2}\left\langle(\boldsymbol{h} \cdot \boldsymbol{p})^{4}\right\rangle
$$

the positive representation

$$
p=\sqrt{2}\left(\begin{array}{llll}
0 & 0 & 0 & 1 \\
1 & 0 & 0 & 0 \\
0 & 1 & 0 & 0 \\
0 & 0 & 1 & 0
\end{array}\right), \quad p^{\prime}=p^{T}
$$

establishes the negativity (non-positivity) of $F_{4}(1,2,3,4)$ for bilinear ferromagnetic Hamiltonians.

For $F_{6}$, with $n=3$, the linear combination $d$ of (10.14) is already sufficient, since $1^{2}+\omega^{2}+\left(\omega^{2}\right)^{2}=0$; thus

$$
F_{6}[\boldsymbol{h} \cdot \boldsymbol{\sigma}]=\frac{1}{3}\left\langle(\boldsymbol{h} \cdot \boldsymbol{d})^{6}\right\rangle,
$$

and with the representation $(10.20), F_{6}(1,2,3,4,5,6)$ is positive for bilinear ferromagnetic Hamiltonians. 


\section{Conclusions}

We have examined two techniques for the bounding of correlations on Ising spin lattices: the product space extension of the lattice configuration space, and the selection of a suitable representation on this space for exhibiting manifest positivity of selected correlations. These techniques seem quite powerful for investigation of correlations of any given order. However, it is not clear that the machinery for investigation of correlations of general order - e.g. $F_{n}$ for arbitrary $n$ - is at hand. Certain quantities of formally infinite order, such as the direct (s-particle irreducible) correlations may already be amenable to the above techniques, but here too the requisite analysis remains to be carried out.

There are two obvious, qualitatively distinct, directions in which one may now proceed, even in the context of correlations of given order: Hamiltoniandependent correlations on lattices, and correlations in quantum systems. The class of inequalities we have been concerned with in the bulk of this paper has been exceedingly general, restricted only to some suitably defined ferromagnetic character of the underlying interaction. It seems clear that far stronger relations would be available if more detailed information on the nature of the coupling could be used. The relevant machinery has in fact been set up - see (3.9), (3.10). There are required only lattice analogs of the sum rules (3.9); these have been found, and the consequent extended inequalities will be presented in a future publication.

Let us turn briefly to the subject of quantum correlation inequalities. Indeed, the writer was led to the formulation of this paper by a study of occupation number correlation inequalities for Fermion fluids, as a needed ${ }^{3}$ companion to those discussed in Section 5. In such a case, the choice of a suitable representation is implicit in the quantum mechanics, and is superfluous as a "technique". The major formal change in the analysis is in the dimensionality of the space at each "site" (read "one-body state index"), coupled with some expected but not intractable commutativity problems. These results will be reported in another publication. However, the analogous collection of correlation inequalities for Boson systems is much more of an open problem.

Acknowledgements. I would like to thank P. Cartier, J. Ginibre, E. Lieb, J. Lebowitz, and C. Newman for fruitful conversations and welcome encouragement.

\section{References}

1. See e.g. Aranoff, S., Percus, J. K.: Phys. Rev. 162, 878 (1967)

2. Kijewski, L. J., Percus, J. K.: Phys. Rev. 164, 228 (1967)

3. See e.g. Percus, J.K.: In: Statistical mechanics, ed. Rice, S., Freed, K., Light,J., p. 337, University of Chicago Press 1972

4. See e.g. Garrod, C., Percus, J. K.: J. Math. Phys. 5, 1756 (1964)

5. Percus, J.K.: Reduced density matrices. Courant Institute Lecture Notes 1969 (unpublished)

6. Mazzaiotti, A., Parr, R. G.: J. Chem. Phys. 52, 1605 (L) (1970)

7. Rosina, M., Percus, J., Kijewski,L., Garrod, C.: J. Math. Phys. 10, 1761 (1969)

8. Yang, C. N.: Phys. Rev. 85, 809 (1952)

9. Griffiths, R.B.: J. Math. Phys. 8, 484 (1967)

10. Griffiths, R. B.: J. Math. Phys. 8, 478 (1967) 
11. Ginibre, J.: Phys. Rev. Letters 23, 828 (1969)

12. See e.g. Ginibre, J.: Mathematical aspects of statistical mechanics, ed. Pool,J.C.T. p. 27. Providence, R. I.: American Mathematical Society 1971

13. See e.g. Fisher, M.: J. Math. Phys. 7, 1776 (1966)

14. Griffiths, R. B.: J. Math. Phys. 10, 1559 (1969)

15. See e.g. Gantmacher,F.R.: Theory of matrices, Vol. II, Chapter XIII. New York: Chelsea Pub. Co.

16. Fortuin, Kasteleyn, P., Ginibre, J., Commun. math. Phys. 22, 89 (1971)

17. Lebowitz, J.: Commun. math. Phys. 35, 87 (1974)

18. Percus, J. K.: Harvard University Seminar, Jan. (1974)

19. Griffiths, R., Hurst, C., Sherman, S., J. Math. Phys. 11, 790 (1970)

20. Cartier,P.: Communicated to the writer by B. Simon

Communicated by G. Gallavotti

J. K. Percus

Courant Institute

of Mathematical Sciences

New York University

New York, N.Y. 10012, USA 